\title{
Excitation of Zonal Flow by Drift Waves in Toroidal Plasmas
}

\author{
Liu Chen \\ Department of Physics and Astronomy, University of California, Irvine CA. 92697 \\ Zhihong Lin, Roscoe White \\ Plasma Physics Laboratory, Princeton University, P.O.Box 451, \\ Princeton, New Jersey 08543
}

\begin{abstract}
An analytical dispersion relation is derived which shows that, in toroidal plasmas, zonal flows can be spontaneously excited via modulations in the radial envelope of a single- $n$ coherent drift wave, with $n$ the toroidal mode number. Predicted instability features are verified by 3D global gyrokinetic simulations of the ion-temperature-gradient mode. Nonlinear equations for mode amplitudes demonstrate saturation of the linearly unstable pump wave and bursting behaviour in the drift-wave intensity and zonal flows.

PACS numbers: 52.35.Kt, 52.35.Ra
\end{abstract}

Recent 3D gyrokinetic [1,2] and gyrofluid [3] simulations in toroidal plasmas have demonstrated that zonal flows [4] play a crucial role in regulating the nonlinear evolution of electrostatic drift-wave instabilities such as the ion temperature gradient (ITG) modes and, as a consequence, the level of the anomalous ion thermal transport. Zonal flows correspond to potentials which spatially depend only on the radius $\mathrm{r}$ and contain temporal variations with time scales longer than that of the drift waves. Recent gyrokinetic simulations [5] have shown that zonal flows could be spontaneously excited by ITG turbulence held at constant level, suggesting parametric instability processes as the generation mechanism. Diamond et. al. [6] have proposed the modulational instability of drift-wave turbulence ("plasmons") in a slab-geometry treatment. Those authors also noted that unstable zonal flows can couple back to the drift waves and proposed a predator-prey model for the nonlinear self regulation of the drift wave turbulence.

In the present letter, we show, both analytically and by direct 3D gyrokinetic simulations, that zonal flows can be readily excited via the modulational instability of a single- $n$ coherent drift wave in toroidal plasmas, with $n$ the toroidal mode number. We note that our theory is strictly applicable 
to toroidal geometry. Specifically, the drift wave, while having only a single $n$ value, contains many poloidal harmonics (m's) which are toroidally coupled. Thus the modulation corresponds to that of the radial envelope describing the magnitude of each poloidal harmonic. In this respect the zonal flow can be regarded as the radial envelope mode.

Consider a large aspect ratio $(\epsilon=a / R<<1)$ tokamak plasma with the usual radial (r), poloidal $(\theta)$ and toroidal $(\phi)$ coordinates. Here $R$ and $a$ are respectively the major and minor radii. Electrostatic fluctuations are taken to be coherent and composed of a single $n(n \neq 0)$ drift wave, $\delta \phi_{d}$ and a zonal flow mode $\delta \phi_{z}$; that is, $\delta \phi_{d}=\phi_{0}+\delta \phi_{+}+\delta \phi_{-}+$c.c.

$$
\begin{gathered}
\phi_{0}(\vec{r}, t)=e^{-i\left(n \phi+\omega_{0} t\right)} \sum_{m} \Phi_{0}(m-n q) e^{i m \theta}, \\
\delta \phi_{ \pm}=e^{i\left(\mp n \phi-\left(\omega_{z} \pm \omega_{0}\right) t+K_{z} r\right)} \sum_{m} \Phi_{ \pm}(m-n q) e^{i m \theta}
\end{gathered}
$$

and $\delta \phi_{z}=\Phi_{z} e^{i\left(K_{z} r-\omega_{z} t\right)}+$ c.c.. Thus $\phi_{0}$ is the pump drift wave and $\omega_{0}$ its eigenmode frequency; $\delta \phi_{+}$ and $\delta \phi_{-}$are respectively the upper and lower sidebands produced by the modulation in the radial envelope due to $\delta \phi_{z}$ at frequency $\omega_{z}$ and radial wavenumber $K_{z}$. We have assumed $n>>1$ and adopted the ballooning mode representation [7] in which $K_{z}=n q^{\prime} \theta_{0}, q=r B_{\phi} / R B_{\theta}$ is the safety factor, and $0 \leq \theta_{0} \leq \pi$ is the Bloch phase shift. The pump mode $\phi_{0}$ has $\theta_{0}=0$, (ie. a flat radial envelope) which is, for a given $n$, usually the linearly most unstable mode. On the other hand $\delta \phi_{+}$ and $\delta \phi_{-}$have $\theta_{0} \neq 0$ giving radial envelope modulations. Typically they are linearly stable for moderate values of $\theta_{0}[3]$. We are thus dealing with a four-wave coupling process among $\phi_{0}, \delta \phi_{+}$, $\delta \phi_{-}$, and $\delta \phi_{z}$. Three wave parametric excitation of zonal flows can be shown to be rather ineffective due to the frequency and wavenumber matching constraints.

Since electrons are adiabatic for the $n \neq 0$ drift waves, only ions contribute to the nonlinear physics. $\delta \Phi_{z}$ is then coupled to $\Phi_{0}$ and $\delta \phi_{ \pm}$, and the nonlinear coupling coefficient is formally of the Hasegawa-Mima type [8-10], i.e. 


$$
\left(-i \omega_{z}+\nu_{z}\right) \chi_{i z} \Phi_{z}=g\left\langle\sum_{m}\left[a_{+} \Phi_{0}^{*} \Phi_{+}-a_{-} \Phi_{0} \Phi_{-}\right]\right\rangle
$$

where $g=\frac{c}{2 B} \alpha_{i} \rho_{i}^{2} k_{\theta} K_{z}, a_{+}=k_{0 \perp}^{2}-k_{+\perp}^{2}, a_{-}=k_{0 \perp}^{2}-k_{-\perp}^{2}, \chi_{i z} \simeq 1.6 \epsilon^{3 / 2} K_{z}^{2} \rho_{i}^{2} B_{\phi}^{2} / B_{\theta}^{2}[11], \nu_{z}=$ $\left(1.5 \epsilon \tau_{i i}\right)^{-1}[12], \vec{k}_{0 \perp}=\hat{\theta} k_{\theta}+i n q^{\prime} \hat{r} \partial_{\zeta}, k_{\theta}=n q / r_{0}, r_{0}$ refers to one reference mode surface, $\zeta=m-n q$ corresponds to the fast radial variable, $\vec{k}_{ \pm \perp}=\hat{r} K_{z} \pm \vec{k}_{0 \perp}, \tau=T_{i} / T_{e}$ and $\langle A\rangle=\int_{-1 / 2}^{1 / 2} A d \zeta$ is an averaging with respect to $r_{0}, \alpha_{i} \simeq \delta P_{0 \perp} /\left(N e \Phi_{0}\right)+1$ and $\delta P_{0 \perp}$ is the perturbed perpendicular pressure due to $\Phi_{0}$. The detailed expression for $\alpha_{i}$ depends on the specific drift wave mode and plasma parameters; e.g. $\quad \alpha_{i} \simeq 1+\tau+\eta_{i} \tau, \eta_{i}=d \ln T_{i} / d \ln N$ for the electron drift wave, and $\alpha_{i} \simeq \tau\left(1+\eta_{i}\right) /\left[(3 \tau-1) L_{n} / R+1 / 2\right]+1$ for ITG in the fluid ion local approximation [3]. Here $L_{n}^{-1}=d \ln N / d r$. In deriving Eq. 3 we have assumed $\left|k_{\perp} \rho_{i}\right|<1$ with $\rho_{i}$ the ion gyro radius, $\left|\omega_{z}\right|<\omega_{G A M}$, and averaged over the Geodesic Acoustic Mode [13].

The nonlinear coupling of $\delta \phi_{ \pm}$to $\Phi_{0}$ and $\delta \Phi_{z}$ can be straightforwardly calculated using the nonlinear gyrokinetic equation $[9,10]$ and the quasi neutrality condition. We have, denoting $\omega_{+}=\omega_{z}+\omega_{0}$,

$$
\begin{array}{r}
\mathcal{L}_{+} \Phi_{+}=\omega_{+}\left[(1+\tau) \Phi_{+}\right. \\
\left.-\left(T_{i} / e\right)\left\langle\int d^{3} \vec{v} J_{0} \delta G_{i+}^{l}\right\rangle\right] \\
=-i(c / B) k_{\theta} K_{z} \tau \Phi_{0}(\zeta) \Phi_{z}
\end{array}
$$

where $\delta G_{i+}^{l}$ satisfies the linear gyrokinetic equation $[14,15]$

$$
\left(v_{\|} \vec{b} \cdot \vec{\nabla}-i \omega_{+}+i \vec{k}_{+\perp} \cdot \vec{v}_{d}\right) \delta G_{i+}^{l}=-h \Phi_{+}
$$

with $h=\left(\omega_{+}-\omega_{* i}\right) F_{M i} J_{0} e / \tau_{i}$ and $\vec{b}=\vec{B}_{0} / B_{0}, \vec{v}_{d}$ is the magnetic $\vec{\nabla} B_{0}$ and curvature drift, $J_{0}=$ $J_{0}\left(k_{+\perp} \rho_{i}\right), \omega_{*}=\omega_{* i n}\left[1+\eta_{i}\left(v^{2} /\left(2 v_{i t}^{2}\right)-3 / 2\right)\right], \omega_{* i n}=k_{\theta} \rho_{i t} v_{i t} / L_{n}$, and $F_{M i}$ is the Maxwellian ion distribution with $v_{i t}$ the ion thermal velocity. $\mathcal{L}_{+}=\mathcal{L}\left(\omega_{+}, \vec{k}_{+\perp}, \zeta\right)$ and $\mathcal{L}$ is just the linear operator for the drift wave eigenmode. In particular, $\mathcal{L}_{0} \Phi_{0}=\mathcal{L}\left(\omega_{0}, \vec{k}_{0 \perp}, \zeta\right) \Phi_{0}=0$ with $\omega_{0}$ the eigenmode frequency. In deriving the nonlinear response in Eq.4 we have assumed fluid ions. Since Eq. 4 depends only on $\zeta$ we can solve it by first Fourier transforming to the along-field-line ballooning coordinate $\eta$. Letting $\Phi_{+}=A_{+} \Phi_{0}(\zeta)$ and $\hat{\Phi}_{0}(\eta)$ equal the Fourier transform of $\Phi_{0}(\zeta)$ we readily find 


$$
A_{+}=-i c k_{\theta} K_{z} \tau \Phi_{z} / B D_{+}
$$

where $D_{+}\left(\omega_{+}, k_{\theta}, K_{z}\right) \equiv\left\langle\left\langle\hat{\Phi}_{0}^{*} \hat{\mathcal{L}}_{+} \hat{\Phi}_{0}\right\rangle\right\rangle /\left\langle\left\langle\left|\hat{\Phi}_{0}\right|^{2}\right\rangle\right\rangle$, with $\langle\langle A\rangle\rangle=\int_{-\infty}^{\infty} d \eta A$, and $\hat{\mathcal{L}}_{+}=\mathcal{L}\left(\omega_{+}, \hat{\theta} k_{\theta}+\right.$ $\left.\hat{r}\left(k_{\theta} \hat{s} \eta+K_{z}\right),-i \partial_{\eta}\right)$ is the corresponding linear drift wave operator in the $\eta$ coordinate, $\hat{s}=q^{\prime} r / q$ the local shear.

Letting $\Omega_{+}=\omega\left(k_{\theta}, K_{z}\right)$ be the eigenfrequency for the upper side band and noting that $\left|\omega_{z}\right|, \mid \Omega_{+}-$ $\omega_{0} \mid<<\omega_{0}, D_{+}$can then be approximated as $D_{+} \simeq\left(\partial D_{0 r} / \partial \omega_{0}\right)\left(\omega_{z}+\Delta+i \gamma_{d}\right)$, where $D_{0 r}$ is the Hermitian part of $D_{0}, \partial D_{0 r} / \partial \omega_{0} \simeq \tau, \Delta=\omega_{0}-\Omega_{+r}$ is the frequency mismatch and and $\gamma_{d}=-\Omega_{+i}$ is the sideband damping rate.

Similar analysis can also be carrried out for the lower sideband. Thus we find $\Phi_{-}=A_{-} \Phi_{0}(\zeta)$,

$$
A_{-}=i c k_{\theta} K_{z} \tau \Phi_{z} / B D_{-}
$$

and $D_{-}=\left(\partial D_{0 r} / \partial \omega_{0}\right)\left(\omega_{z}-\Delta+i \gamma_{d}\right)$. Here $\Omega_{+}=\Omega_{-}=\omega\left(k_{\theta},-K_{z}\right)$ due to the up-down symmetry. Substituting $\Phi_{ \pm}=A_{ \pm} \Phi_{0}$ into Eq. 3 and noting that $\left\langle\sum_{m}\left|\Phi_{0}\right|^{2}\right\rangle=\int_{-\infty}^{\infty}\left|\Phi_{0}\right|^{2} d \zeta=\left\langle\left\langle\left|\hat{\Phi}_{0}\right|^{2}\right\rangle\right\rangle$ we finally obtain the desired linear dispersion relation for the modulational instability

$$
\Gamma_{z}+\nu_{z}=\gamma_{M}^{2}\left(\Gamma_{z}+\gamma_{d}\right) /\left[\Delta^{2}+\left(\Gamma_{z}+\gamma_{d}\right)^{2}\right]
$$

where we have let $-i \omega_{z}=\Gamma_{z}$ and $\gamma_{M}^{2}=\left(\alpha_{i} / 1.6 \epsilon^{3 / 2}\right)\left(B_{\theta} k_{\theta} c_{s} K_{z} \rho_{s} / B_{\phi}\right)^{2}\left\langle\left\langle\left|e \hat{\Phi}_{0} / T_{e}\right|^{2}\right\rangle\right\rangle$. With appropriate $\alpha_{i}$ Eq. 8 is valid for various branches of drift waves such as the electron drift wave or ITG. It can be solved for $\Gamma_{z}$ in two limits. In the $|\Delta|<\gamma_{d}, \gamma_{M}$ limit, we have

$$
\Gamma_{z}=-\left(\gamma_{d}+\nu_{z}\right) / 2+\left[\gamma_{M}^{2}+\left(\gamma_{d}-\nu_{z}\right)^{2} / 4\right]^{1 / 2}
$$

Thus while the instability has a threshold at $\gamma_{M}^{2} \simeq \nu_{z} \gamma_{d}$, strong growth with $\Gamma_{z} \simeq \gamma_{M}$ only sets in when $\gamma_{M} \gtrsim \gamma_{d} / 2$. On the other hand for $\gamma_{M},|\Delta|>\gamma_{d}, \nu_{z}$ we have

$$
\Gamma_{z} \simeq \gamma_{M}\left(1-\Delta^{2} / \gamma_{M}^{2}\right)^{1 / 2} .
$$


Again, strong growth with $\Gamma_{z} \simeq \gamma_{M}$ sets in when $\gamma_{M} \gtrsim|\Delta|$. Note that for $\Gamma_{z} \simeq \gamma_{M} \propto\left|K_{z} \rho_{s}\right|\left|e \hat{\Phi}_{0} / T_{e}\right|$, the coherent modulational instability is generally much stronger than that of the drift wave "plasmons" with growth rate $\propto\left|e \hat{\Phi}_{0} / T_{e}\right|^{2}$. Furthermore, $|\Delta| \simeq K_{z}^{2}\left(\partial D_{0 r} / \partial k_{0 \perp}^{2}\right) / 2\left(\partial D_{0 r} / \partial \omega_{0}\right) \simeq$ $K_{z}^{2} \rho_{s}^{2}(1+\tau) / 2$, and $\gamma_{d}$ generally increases with $K_{z} \rho_{s} . \Gamma_{z}$, for a given $\left|\hat{\Phi}_{0}\right|$, can then be expected to first increase with $K_{z} \rho_{s}$ but eventually decrease at large $K_{z} \rho_{s}$. While the exact $\left(K_{z} \rho_{s}\right)_{m}$ at which $\Gamma_{z}$ maximizes can not be predicted, it in general will increase with the pump wave amplitude $\left|\hat{\Phi}_{0}\right|$. Thus far away from linear marginal stability one expects strong linear instability, larger $\left|\hat{\Phi}_{0}\right|$ and modulational instabilities peaked around Bloch phase shifts $\theta_{0 m} \sim O(1)$, ie $\left(K_{z} \rho_{s}\right)_{m} \sim k_{\theta} \rho_{s} \hat{s}$. That is, with strong linear drive the radial scale lengths of zonal flows and drift wave envelopes should be on the order of a typical distance between adjacant mode rational surfaces.

The predicted modulational instability features have been observed in 3D global gyrokinetic simulations of ITG modes using the gyrokinetic toroidal code [2]. These nonlinear simulations keep only a single toroidal mode $n \neq 0$ initially. The starting fluctuation level is very low to allow linear ITG eigenmode structure to be formed before nonlinear saturation. When the ITG mode grows to a desired amplitude, an external damping is applied so that the mode amplitude stays constant. Zonal flow with a single radial mode number is now self-consistently included. We observe exponential growth of zonal flow until it reaches a high level where the ITG mode is suppressed. The radial envelope modulation of the ITG mode correlates with the zonal flow radial structure. As shown in Fig. 1 (A), the growth rate of zonal flow with a fixed radial mode number linearly depends on the ITG mode amplitude except at large amplitude where ITG nonlinear effects appear. Analytical prediction of zonal flow growth rate from the solution of Eq. 9, is shown by the solid line in Fig. 1 (A). In the analytical calculation, the sideband damping rate is estimated from simulations to be $\gamma_{d} \sim 1.5 \gamma_{0}$ including both intrinsic damping and externally-applied damping, and $\gamma_{0}$ is the pump ITG intrinsic linear growth rate. For a fixed ITG mode amplitude, measured zonal flow growth rate 
increases linearly with radial mode number $\left(\right.$ or $\left.\theta_{0}\right)$ for small $\theta_{0}$ and decreases for large $\theta_{0}$, as shown in Fig. 1 (B), consistent with theory.
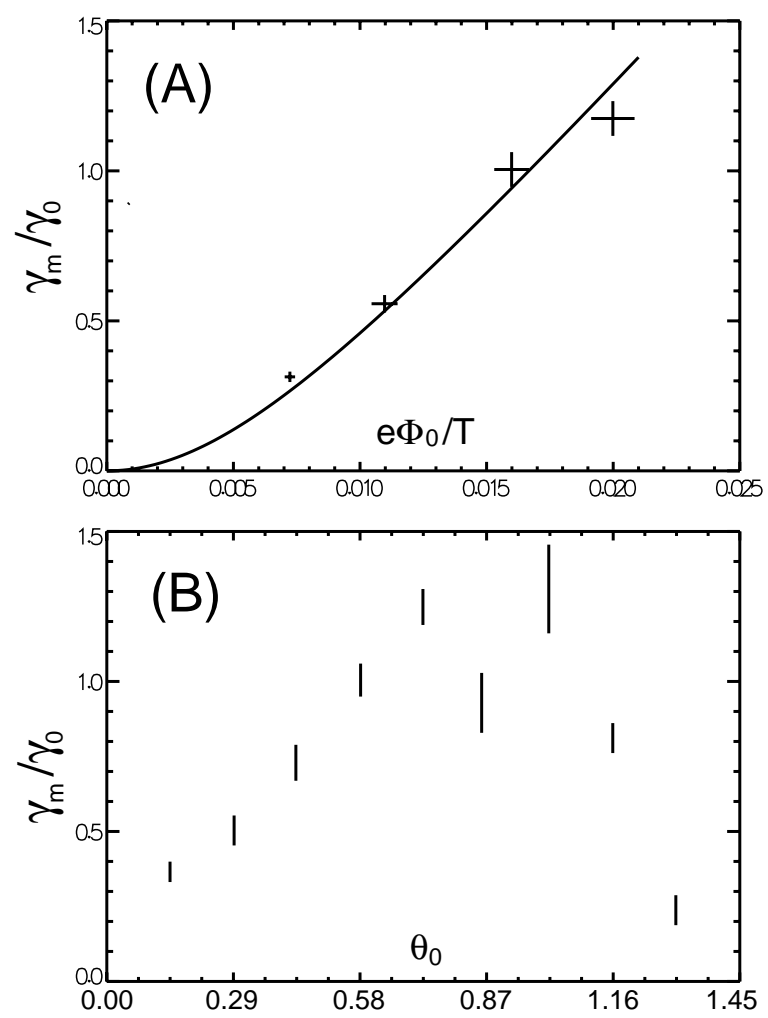

FIG. 1. Gyrokinetic simulation results of zonal flow growth rate $(A)$ vs. ITG mode amplitude for fixed $\theta_{0}$, and $(B)$ vs. $\theta_{0}$ for fixed ITG amplitude, normalized to ITG growth, $\gamma_{0}$. The line in (A) is the solution of Eq. 9.

We now consider the nonlinear evolution of this modulation instability. As $\delta \phi_{z}$ and $\delta \phi_{ \pm}$exponentiate in amplitude, they will nonlinearly couple and induce damping in the pump wave amplitude. Replacing $\omega_{0}$ by $\omega_{0}+i \partial_{t}$, letting $\left\langle\left\langle\left|e \hat{\Phi}_{0} / T_{e}\right|^{2}\right\rangle\right\rangle=A_{0}^{2}$ and including the linear growth rate $\gamma_{0}$ the equation for $A_{0}(t)$ becomes

$$
\left(\frac{d}{d t}-\gamma_{0}\right) A_{0}=-\frac{c T_{e}}{e B} \frac{\tau k_{\theta} K_{z}}{\partial D_{0 r} / \partial \omega_{0}}\left(A_{-} \Phi_{z}+A_{+} \Phi_{z}^{*}\right)
$$

Equations governing $A_{+}, A_{-}=A_{+}^{*}$ and $\Phi_{z}$ are given by Eqs. 3,4,6 and noting that $D_{ \pm} \simeq$ $\partial D_{0 r} / \partial \omega_{0}\left(d / d t \mp i \Delta+\gamma_{d}\right)$. Using dimensionless time $\tau=\gamma_{0} t$ and performing straightforward normalizations such that $A_{0} \propto P, A_{+} \propto S e^{i \Psi(t)}$, and $\Phi_{z} \propto Z$, we find

$$
\frac{d P}{d \tau}=P-2 Z S \cos (\Psi)
$$




$$
\begin{gathered}
\frac{d S}{d \tau}=-\Gamma_{d} S+Z S \cos (\Psi) \\
\frac{d Z}{d \tau}=-\gamma_{z} Z+2 P S \cos (\Psi) \\
\frac{d \Psi}{d \tau}=\delta-\frac{P Z}{S} \sin (\Psi)
\end{gathered}
$$

with $\Gamma_{d}=\gamma_{d} / \gamma_{0}, \gamma_{z}=\nu_{z} / \gamma_{0}$, and $\delta=\Delta / \gamma_{0}$. These equations are similar to those for three-wave coupling [16], hence we anticipate similar behaviour, such as the existence of a stable attractor and a period doubling route to chaos.

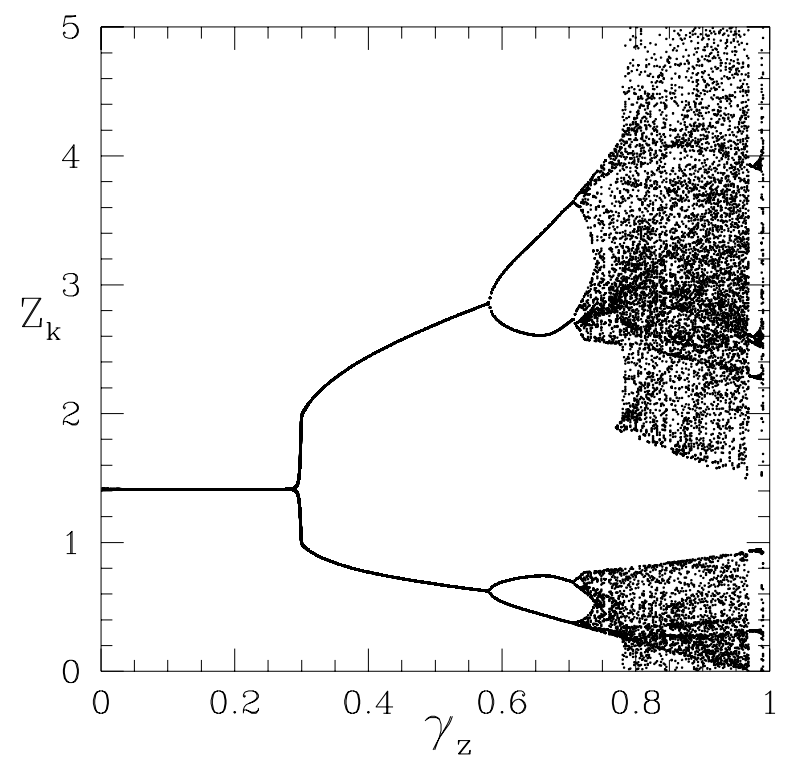

FIG. 2. Values of $Z_{k}, \delta=2, \Gamma_{d}=2$

Introduce an associated one dimensional map by defining times $\tau_{k}$ at successive zeros of $d Z / d \tau$. Numerical plot of the values of $Z_{k}$ in steady state (after transients have died away) is shown in Fig. 2 for $\delta=2, \Gamma_{d}=2$. Eqs. $12-15$ have a fixed point attractor for $\gamma_{z} \lesssim 0.3$. For $0.3 \lesssim \gamma_{z} \lesssim 0.58$ the attractor is a stable limit cycle with the bounding values of $\mathrm{Z}$ given by the two branches in Fig. 2. The initial bifurcation of the stable fixed point into the limit cycle corresponds also to period doubling, as can be seen in the plot of associated frequencies, Fig. 3. The two frequencies correspond to a rapid increase in $\mathrm{Z}$ to the upper value followed by a slow decay to the lower. Damping to the 
fixed point, obtained below analytically, is also shown. Apparent chaos sets in for $\gamma_{z} \gtrsim 0.75$.

The fixed point of Eqs $12-15$ is readily found to be $Z_{0}=\sqrt{\left(\delta^{2}+\Gamma_{d}^{2}\right) /\left(2 \Gamma_{d}\right)}, P_{0}=\sqrt{\gamma_{z}} Z_{0}, S_{0}=$ $P_{0} / \sqrt{2 \Gamma_{d}}, \sin \Psi_{0}=\delta / \sqrt{\delta^{2}+\Gamma_{d}^{2}}$. Linearizing Eqs. 12-15 about the fixed point we find for the complex frequency

$$
\omega^{4}-i c_{3} \omega^{3}+c_{2} \omega^{2}-i \omega c_{1}+c_{0}=0
$$

with $c_{0}=4 \gamma_{z}\left(\Gamma_{d}^{2}+\delta^{2}\right), c_{1}=4 \gamma_{z} \Gamma_{d}+4 \gamma_{z} \delta^{2} / \Gamma_{d}, c_{2}=\gamma_{z} \delta^{2} / \Gamma_{d}-\gamma_{z} \Gamma_{d}-\delta^{2} / \Gamma_{d}+\Gamma_{d}-\Gamma_{d}^{2}-\delta^{2}$, $c_{3}=1-2 \Gamma_{d}-\gamma_{z}$

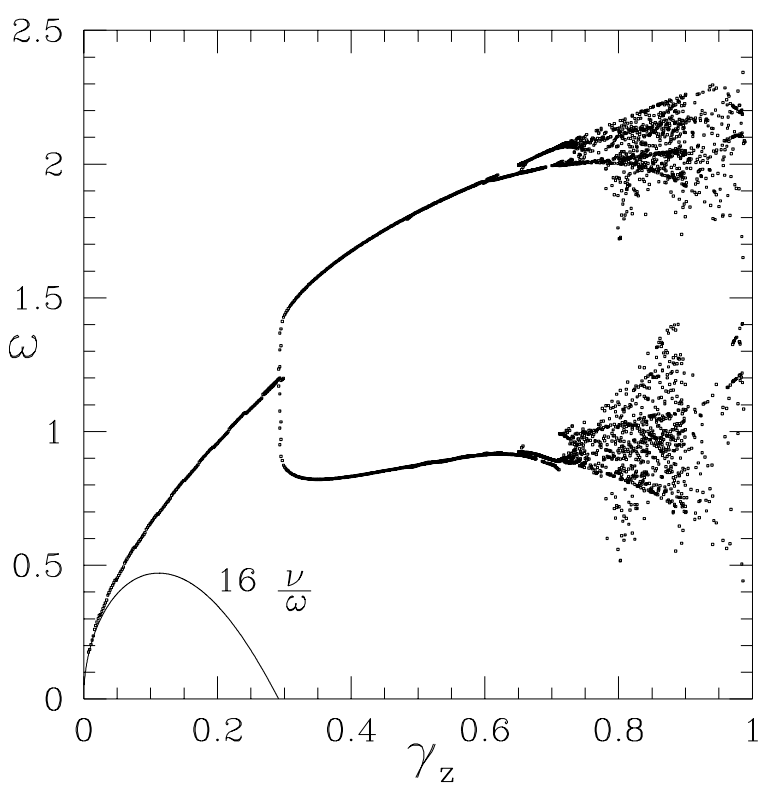

FIG. 3. Frequencies $(\omega=2 \pi f)$ for map, $\delta=2, \Gamma_{d}=2$, and damping $\nu$ for approach to the fixed point.

Destabilization of the fixed point is obtained by requiring that the frequency be real, giving $c_{1} c_{2} c_{3}=$ $c_{1}^{2}+c_{0} c_{3}^{2}$ with the frequency given by $\omega^{2}=-c_{1} / c_{3}$. In Fig. 4 is shown the domain in which the stable fixed point exists. The boundaries of the stable domain at $\gamma_{z}=0$ are given by $\Gamma_{d}=1 / 2$ and $\Gamma_{d}^{3}=\delta^{2}\left(\Gamma_{d}+1\right)$. For small $\gamma_{z}$ the real frequency and the damping behave as $\omega \simeq A \sqrt{\gamma_{z}}, \nu \simeq B \gamma_{z}$, with $A=2 \sqrt{\left(\Gamma_{d}^{2}+\delta^{2}\right) /\left(\Gamma_{d}^{2}+\delta^{2}-\Gamma_{d}+\delta^{2} / \Gamma_{d}\right)}$ and $B=2\left(2 \delta^{2}-\Gamma_{d}^{3}-\Gamma_{d} \delta^{2}\right) /\left(\Gamma_{d}^{2}+\delta^{2}-\Gamma_{d}+\delta^{2} / \Gamma_{d}\right)^{2}$. The ratio of damping to frequency is shown in Fig. 3. 


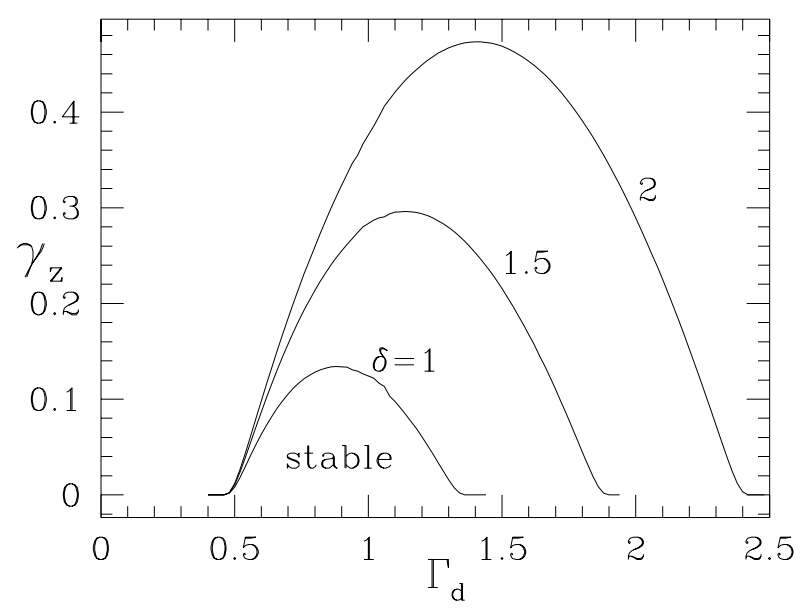

FIG. 4. Destabilization of the fixed point.

Present turbulence simulations have $\Gamma_{d} \sim 1$, with values of $\gamma_{z}$ and $\delta$, placing them in the stable fixed point domain. The oscillations observed are thus probably nonlinear transient decay to the fixed point, with the decay time much longer than the simulation time. The drift wave intensity is $I_{d}=P_{0}^{2}+2 S_{0}^{2}$. Assuming weak turbulence scaling of $\chi_{i} \propto I_{d}$, where $\chi_{i}$ is the anomalous ion thermal transport coefficient, we find that in the stable domain $\chi_{i} \propto \gamma_{z} \propto \nu_{i i}$ consistent with the trend observed in simulations [5]. In the future we will explore the route to chaos with nonlinear simulations and examine its implications for $\chi_{i}$. Finally, we note that, assuming nonlinear interactions among $n \neq 0$ toroidal drift modes are ignorable, the present results, obtained for a single-n mode, can be readily generalized to a spectrum of multiple-n toroidal modes.

\section{ACKNOWLEDGMENTS}

This work was supported by the U.S. Department of Energy Grant DE-FG03-94ER54271 and under contract number DE-AC02-76-CHO3073. The authors acknowledge useful discussions with M. A. Beer, P. H. Diamond, T. S. Hahm, F. L. Hinton, and F. Zonca. 
[1] A. M. Dimits et al., Phys. Rev. Lett. 77, 71 (1996).

[2] Z. Lin, T. S. Hahm, W. W. Lee, W. M. Tang, and R. B. White, Science 281, 1835 (1998).

[3] M. A. Beer, PhD dissertation, Princeton University, 1995.

[4] A. Hasegawa, C. G. Maclennan, and Y. Kodama, Phys. Fluids 22, 2122 (1979).

[5] Z. Lin, T. S. Hahm, W. W. Lee, W. M. Tang, and P. H. Diamond, Phys. Rev. Lett. 83, ??? (1999).

[6] P. H. Diamond, et al., in Proceedings of the 17th IAEA Conference on Controlled Fusion and Plasma Physics, Yokahama, Japan, 1998.

[7] J. W. Connor, R. J. Hastie and J. B. Taylor, Proc. Royal Soc. 365, 1 (1979).

[8] A. Hasegawa and K. Mima Phys. Rev. Lett. 39, 205 (1977).

[9] E. A. Frieman and L. Chen, Phys. Fluids 25, 502 (1982).

[10] F. L. Hinton, Private communication.

[11] M. N. Rosenbluth and F. L. Hinton, Phys. Rev. Lett. 80, 724 (1998).

[12] F. L. Hinton and M. N. Rosenbluth, Plasma Phys. Controlled Fusion 41, A653 (1999).

[13] N. Winsor et al., Phys. Fluids 11, 2448 (1968).

[14] P. H. Rutherford and E. A. Frieman, Phys. Fluids 11, 569 (1968).

[15] J. B. Taylor and R. J. Hastie, Plasma Phys. 10, 479 (1968).

[16] J. M. Wersinger, J. M. Finn and E. Ott, Phys. Rev. Lett. 44, 453 (1980). 